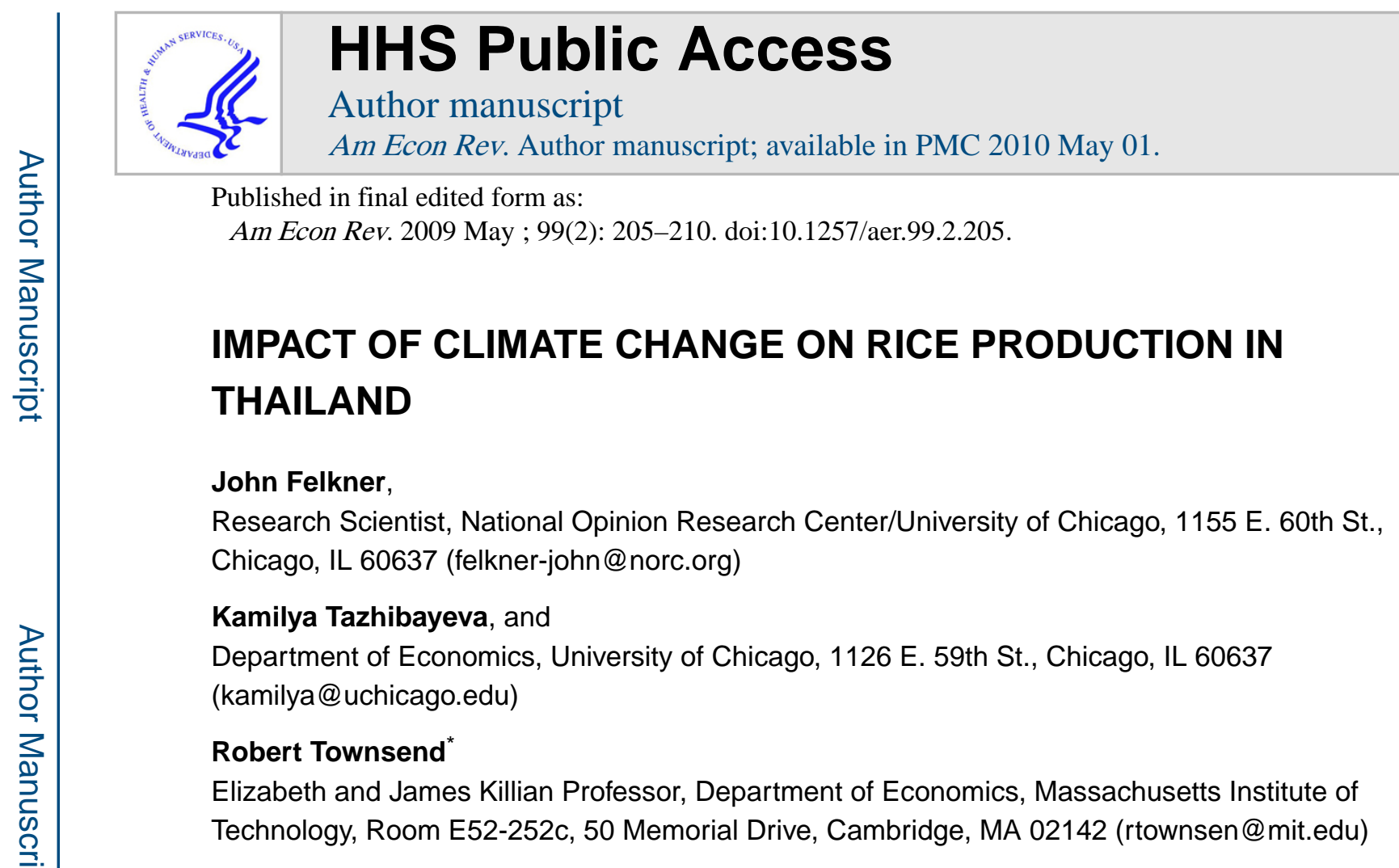

Our goal is to evaluate crop yield impacts from likely climate changes for Southeast Asia. To do so we link soil science crop modeling, weather simulators, and global climate change modeling into an integrated economic model of multi-stage rice production. The economic model is estimated with detailed monthly data on inputs, operations, and environmental data over a five year period. We then forecast impacts under two different future economic scenarios, one assuming high future global anthropogenic pollution emissions, and the other assuming low. We compare results of the integrated economic model with those of a biophysical model, inputting into both the stochastic realizations of a weather generator, calibrated against the present, no climate benchmark and against the two climate mild and severe climate change scenarios. The more realistic forecasts from socio economic model thus include important farmer behavioral/mitigation strategies. We discuss both aggregate/ average impacts as well heterogeneity in response.

\title{
I. Modeling Rice Cultivation
}

Crop cultivation is defined by the process of a crop's biological growth. This biological growth consists of distinct, chronologically sequential phases. A crop's need for and responsiveness to a given physical input varies across different growth phases. Depending on the progress of crop growth, the farmer may want to adjust the amounts and types of physical inputs. The farmer responds to realized production shocks as captured in the state of the crop while forecasting future shocks and actions. For rainfall, the history up to a given stage is predictive of the future. Other idiosyncratic shocks are not observed. Thus if using an aggregate single-stage production function, there would be a bias in estimation, that is,

Corresponding Author: Robert Townsend, Department of Economics, Massachusetts Institute of Technology, Room E52-252c, 50 Memorial Drive, Cambridge, MA 02142; rtownsen@mit.edu; telephone: 617-452-3722; fax: 617-253-1330.

Session Chair: Michael Greenstone, Massachusetts Institute of Technology. Discussants: Peter Klenow, Stanford University; Andrew

Foster, Brown University; Douglas Almond, Columbia University. 
production shocks influencing inputs in previous stages are not seen by the econometrician and end up in the overall error term.

We thus use a multi-stage rice production function and identify three growth phases of biological development of the rice plant, roughly planting, growing, and harvesting, and map these into economic production stages by matching the timing of production operations to the timing of plant development1.

Let $i$ index the three production stages and $L_{i}$ and $K_{i}$ denote, correspondingly, labor and capital and other inputs in stage $i$. Let $y_{i}$ be output of stage $i$, with $y_{0}$ describing initial conditions of production such as plot characteristics. Let $e_{i}$ be production shock realized during stage $i$. Then output in stage $i$ is $y_{i}=f_{i}\left(y_{i-1}, L_{i}, K_{i}\right) \exp \left(e_{i}\right)$, for $i=1,2,3$, where $f_{i}$ is stage $i$ - specific Cobb-Douglas production function2.

Again, input decisions are made at the start of each stage, after output from the previous stage is observed, before production shocks for the starting stage are realized, and with updated expectations based on history at that point in time. At the beginning of each stage $i$, farmer maximizes expected profits:

$$
\operatorname{Max}_{L_{i}, K_{i}} E_{i}[\pi]=p E_{i}\left[y_{3}\right]-\sum_{j=i}^{3}\left(w_{j} L_{j}+r_{j} K_{j}\right)
$$

where $p, w_{i}$ and $r_{i}$ are prices of, respectively, final output, and stage $i$ labor and capital inputs. 3

Note that the marginal product of all intermediate inputs captures both their immediate direct effect on the state of the crop at the end of the stage and future indirect effects (through levels of future inputs). To illustrate this point, consider the first order condition with respect to $L_{i}$, where $i<3$ :

$$
p \frac{\partial E_{i}\left[y_{3}\right]}{\partial L_{i}}=w_{i}+\sum_{j=i+1}^{3}\left(w_{j} \frac{\partial E_{i}\left[L_{j}\right]}{\partial y_{i}} \frac{\partial y_{i}}{\partial L_{i}}+r_{j} \frac{\partial E_{i}\left[K_{j}\right]}{\partial y_{i}} \frac{\partial y_{i}}{\partial L_{i}}\right)
$$

The marginal cost on the right hand side incorporates change in future expenses on inputs in stages $j>i$ that will be caused by adjustment of optimal levels of stage $j$ inputs with respect to change in levels of stage $i$ inputs actually used.

Felkner, Tazhibayeva, and Townsend (2009) estimate this three-stage rice production function; see details there. To account for endogeneity of input decisions, they estimate the composite production function and input decision rules as a system of simultaneous

\footnotetext{
${ }^{1}$ Within each stage, several operations can be performed simultaneously.

2 Values of inputs, outputs and production shocks are plot-specific. Plot indexing is omitted for simplicity of presentation. We use the Cobb Douglas but will explore other specifications in future work.

${ }^{3}$ Assume the farmer knows all current and future input prices for a given growing season, as well as final output price. Household production separates from consumption and labor supply when markets are complete and there is some evidence for this in the Townsend Thai monthly data.
} 
equations, making use of stage- and operation-specific input prices as well as observed rainfall and farmer-specific rainfall expectations4. At each stage, inputs are determined simultaneously and also depend on intermediate output, or crop state, from the previous stage, farmer's expectation of production shocks, and input prices. The simultaneous system approach delivers estimates of the parameters of the composite production function and decision rules for all production inputs.

\section{Data}

We use Townsend Thai Project data5 on rice farmers in four villages in Sisaket province, located in rural and poor north-eastern part of Thailand. Data are collected monthly on household-plot level, with many households cultivating several plots in a given year. We use an unbalanced five-year panel on 137 households, with a total of 1,030 crop observations over five years. The data include information on usage and cost of labor, equipment and other non-labor inputs used in separate production operations. We also have sets of measures of plot soil quality, some household socio-economic characteristics, and environmental data such as daily rainfall and the chemical composition of water sources. 6

Monthly plot observations enable us to use plot-specific timing and duration of stages. That is, because of the variation in timing and duration of individual stages and of the overall production cycle across plots, aggregate production shocks such as rainfall have differing effect on different plots because they may hit these plots during different production stages. Similarly, rainfall expectations vary across plots.

To construct a measure of intermediate outputs, we use DSSAT, a powerful computer crop growth model7. DSSAT takes in amounts and timing of application of non-labor and nonequipment production factors such as seeds and fertilizer, as well as detailed data on inherent soil quality and climatic conditions. It then employs physical and biophysical models of soilplant-atmosphere interactions to simulate, day by-day, the biological growth of the plant by computing crop-specific growth responses, measured precisely in laboratory conditions, to physical inputs and changes in soil water, carbon and nitrogen. DSSAT tracks plant's growth with thirty dynamic indicators such as number of leaves per stem, root density, stem weight, etc. This allows us to use DSSAT simulations as estimates of intermediate outputs. 8

Note that DSSAT does not take into account labor inputs or idiosyncratic shocks. DSSAT simulations are thus only approximations of the crop state that should occur under observed soil parameters, climatic conditions and crop inputs, without accounting for human input

\footnotetext{
${ }^{4}$ To clarify, while we perform very detailed analysis to incorporate heterogeneous timing of stages and thus inputs application across plots and cycles, as described in the next section, we do not endogenize timing decisions but treat them as predetermined. This is a margin of adjustment which may matter and further mitigate climate change effects.

5 Detailed description of the project can be found at (Thailand Database Research Archive 2009).

${ }^{6}$ Environmental data were collected from five stations per village. Each plot was linked to the closest station. For details, see Felkner, Tazhibayeva and Townsend (2009). We also use historic rain data for 1970-2000 from national province stations.

${ }^{7}$ Decisions Support System for Agrotechnology Transfer (DSSAT) has been maintained and supported by the International Consortium for Agricultural Systems Applications (ICASA).

${ }^{8}$ In practice, integration of DSSAT with economic model produces mixed results, good for stratified subsamples only. We continue to work to improve the statistical significance.
} 
and idiosyncratic environmental stresses. Thus, DSSAT typically over-predicts actual crop states.

\section{Climate Change Impact Modeling}

For this study, we have chosen to use climate change predictions produced for the $4^{\text {th }}$ Assessment Report of the United Nation's Intergovernmental Panel on Climate Change (IPCC9), released in 2007 (Rex Victor Cruz et al. 2007). We use an "ensemble-mean"10 output of multiple, internationally reputable coupled Atmospheric-Oceanic General Circulation Models (AOGCMs) to produce predicted changes for the Southeast Asia region for the time period 2040-2069, relative to the 1960-1990 baseline period11.

Because of the uncertainty in future anthropogenic global emissions (which may differ dramatically due to economic development, policy decisions or technology changes), as well as to assess the range of likely possible climate changes and impacts, we simulated two alternative economic scenarios selected from a set of widely-used scenarios developed for the IPCC $3^{\text {rd }}$ Assessment Report: the Special Report on Emissions (SRES) A1F1 (highest emissions trajectory scenario) and the B1 (lowest emissions trajectory scenario) (N. Nakicenovic et al. 2000)12, both for the 2040-2069 time period. We did not specifically model El Nino impacts, as our primary focus was on impacts and adaptations to longer-term "baseline" changes.

According to IPCC ensemble-mean predictions, results predicts a net annual increase in temperature of between $1.32^{\circ} \mathrm{C}$ (lowest emissions scenario B1) and $2.01{ }^{\circ} \mathrm{C}$ (highest emissions scenario A1F1) and an increase in annual precipitation of $2.25 \%$ (lowest emissions) and1.0\% (highest emissions) for the 2040-2069 period, relative to the baseline 1961-1990 period (R. V. Cruz et al. 2007).

Assessing the impact of these changes on future agricultural outputs and crop yields is complex, as yields are a result of interactions between temperature precipitation effects, direct physiological effects of increased $\mathrm{CO} 2$, and effectiveness and availability of adaptations (M. L. Parry et al. 2004). Consequently, predictions for Asia are mixed. Some studies find decreases in rain-fed crops in South and South-East Asia (C. Rosenzweig et al. 2001). Others such as Cruz et al. (2007), using the HadCM2 global climate model indicate that crop yields could likely increase up to $20 \%$ in East and South-East Asia, while (Parry et al. 2004) find both increases and decreases in yields for Thailand depending on $\mathrm{CO} 2$ regimes.

\footnotetext{
${ }^{9}$ See the IPCC website for more information.

10"Ensemble-mean" predictions are the mean output from multiple models, run together to avoid potential bias or flaws inherent in any particular climate change model, providing a superior delineation of the forced climate change signal from the natural background variability of the system (F. Giorgi and L. O. Mearns 2002).

${ }^{11}$ The models are listed on the IPCC website.

12 The SRES scenarios, as with all economic scenarios of emissions and their reliability, are a source of some controversy. For example, the SRES scenarios have been criticized, including for their use of Market Exchange Rates (MER) for international comparison, in lieu of theoretically favored PPP exchanges rates, which correct for differences in purchasing power. However, for this micro-study, we accept these scenarios as given.
} 
The integrated approach began by running DSSAT to simulate rice growth for 1,030 individual crop-plots in northern Sisaket province. The DSSAT predictions were positively and significantly correlated with yield variation across the plots for those years, with a statistically significant correlation coefficient of .09. These initial simulations allowed calibration of DSSAT and confirmed its usefulness in capturing crop growth.

Next, the economic model was estimated, using the original plot data from the larger sample. Actual rain data from the longer time series of historical data were used to construct farmer's rain expectations. DSSAT predictions from the first step were used to construct measures of intermediate output from stages one and two.

Next, we simulated future "synthetic" weather from the widely-used WGEN weather simulation model (C. W. Richardson 1981)13, generating 100 stochastic weather year realizations based directly on the statistics computed for the historical, 1972-2003 observed weather data. We refer to these weather realizations as describing a "neutral" scenario, assuming that future climate will be a direct, linear extension of the late 20th century. To generate future weather with SRES climate change scenarios, we inputted future changes to monthly precipitation and temperature and drew 100 realizations for each scenario.

The final step was to use the three generated weather scenarios together with corresponding DSSAT crop simulations as inputs into the estimated economic model to predict yields. For each plot, individual rain expectations were constructed for each set of weather realizations for each scenario. Similarly, measures of intermediate stage one and stage two outputs were constructed from weather realization-specific DSSAT simulations for a given plot. Inputs and final output are then predicted by the economic model. These estimates reflected variation in input usage due to both differences in weather realizations and adjustments made by the farmer in each stage.

\section{Results}

Of the two climate change scenarios we consider, the high emissions scenario is more extreme. While daily temperatures increase under both scenarios, the magnitude of increase under high emissions climate relative to low emissions is about $40 \%$ higher. Daily precipitation increases throughout the year under low emissions climate, with particularly high increase of 5.3\% in December and 4.4\% in January. However under high emissions scenario there is less rain in the second half of the year, starting in June, which is exactly the period of rice cultivation. Thus low emissions climate change brings moderate increase in temperature and more rain, while high emissions climate bodes both higher increase in temperature and less rain for rice cultivation 14 .

\footnotetext{
${ }^{13}$ The WGEN weather generation model (specific details can be found in Richardson 1981) begins by first calculating an extensive set of statistical parameters describing the observed, historical 1972-2003 daily weather data, including mean monthly amounts for all key input variables, as well as including probabilities of wet days, probabilities of dry days, and within-year precipitation variation. WGEN then generates daily values for precipitation, maximum and minimum air temperature and solar radiation for an $\mathrm{N}$-year period at a given location. The precipitation component of WGEN is a Markov-chain--gamma-distribution model. The occurrence of wet or dry days is generated with a first-order Markov-chain model in which the probability of rain on a given day is conditioned on whether the previous day was wet or dry.
} 
DSSAT predicts decrease in aggregate yields compared to the neutral climate simulations for both high and low emissions scenarios by $3.53 \%$ and $13.79 \%$, respectively. This result is highly statistically significant. Lower aggregate yields under low emissions compared to high emissions scenario, despite the fact that low emissions scenario is less extreme of the two, may be due to the damaging effect on the crop of higher rainfall during the final production stage in December and January, when grain is mature and harvesting takes place15. The same results hold for model predictions, although under model predictions the difference in yield decreases under high and low emissions scenarios is much smaller, with respective decrease of $10.81 \%$ and $12.04 \%$.

Table 1 provides plot-level analysis of yield changes, for both DSSAT and economic model predictions. The first three rows of Table 1 compare predicted yields, measured in kilograms per acre, when shifting from neutral to high emissions climate. For each plot, we test the equality of mean yields under neutral and high emissions climates. We then compute the percent of plots that have statistically significant change in yields16. These numbers are reported in the first row of Table 1 . The second row reports the actual size of mean yields change over plots conditioned on the change being statistically significant, and third row expresses this change in percent. In the same manner, rows four to six compare predicted yields when shifting from neutral to low emissions climate, and rows seven to nine compare yields when shifting from low to high emissions climate.

DSSAT predicts lower yields for about a third of the plots under both the low and high emission scenarios. For these plots, decrease in yields is severe, ranging from 30 to $40 \%$. Decrease in yields is stronger when shifting to low emissions scenario, under which both more plots are affected and the scale of yield decrease is higher. DSSAT predictions thus suggest that, despite the fact that high emissions climate has less rain during the second half of the year while low emissions climate has moderately more rain throughout the year, farmers fare worse in low emissions climate.

Model predictions stand in stark contrast with DSSAT predictions. The fraction of the sample experiencing statistically significant yield decrease under high emissions climate more than doubles compared to DSSAT. Yields go down for $68 \%$ of the plots, with the average decrease of about $13 \%$. However, under the low emissions climate yields actually increase for over $80 \%$ of the plots, albeit only by half a percent. Thus, farmers manage to take advantage of the moderate increase in rainfall under the low emissions climate. At the same time, however, there is a chance of complete crop failure, as is the case for a small number of plots.

To check for connection between yield changes and household income, we compute the probability of a household's per capita income being below the median17 given that the

\footnotetext{
${ }^{14}$ Yearly averages of daily change in temperature and precipitation are, respectively, $4.4 \%$ and $2.3 \%$ for low emissions scenario, and $6.8 \%$ and $0.5 \%$ for high emissions scenario. Monthly comparisons are available in the Web appendix (available at http:// www.aeaweb.org/articles.php?doi=10.1257/aer.99.2.205).

${ }^{15}$ This result may be due to interactive effects between temperature, precipitation and CO2 levels: Parry et al. (2004) also found that crop yields also decreased more under the B1 than A1F1 scenario under $\mathrm{CO} 2$ effects.

${ }^{16}$ Table 1 reports results for 5\% significance level. Our results hold at both 1 and $10 \%$ significance levels. Corresponding tables are in the web appendix.
} 
household experienced statistically significant increase (decrease) in yields. We also use two measures of soil quality, $\mathrm{pH}$ level and cation exchange capacity (CEC), to test for differences in soil quality between plots with and without statistically significant yield changes. We find that soil quality is not associated with yield changes no matter which climate change is considered. This is true for both DSSAT and model predictions. This result is intuitive. Soil quality is already taken into account in yield predictions for each climate, and should have no further unexplained effect on farmer's productivity. Household income also does not correlate with yield changes, with one notable exception. The few plots that experience crop failure under low emissions climate according to model predictions have a 70\% chance of having per capita income below median. This result suggests that poorer households are incapable of coping with the moderate climate change that other farmers are able to adjust to18.

Comparison of DSSAT and model predictions illustrates the scope of farmers' ability to adjust to climate change19. For the shift from neutral to low emissions climate, without input adjustments, we see statistically significant yield decrease of large magnitude in a third of our sample plots. Once farmer's responses to climate change are incorporated, the majority of plots do not experience yield decreases and even enjoy a slight increase in yields. Farmers are thus able to adjust to climate change from neutral to low emissions scenario.

For the shift from neutral to high emissions climate, without input adjustments we see statistically significant yield decrease of around $30 \%$ in a quarter of our sample. Once farmer's responses to climate change are incorporated, the fraction of the sample experiencing yield decrease more than doubles, but the magnitude of the decline in average yields decreases by more than half. Farmers thus respond to this more severe climate change with adjustments that prevent large crop failures, at the cost of reducing their yields by about $13 \%$. In other words, farmers are unable to fully neutralize the effects of the more severe climate change. However, by adjusting their crop cultivation routine they are able to mitigate the adverse effects. Our results thus suggest that various climate changes pose different challenges to the farmers. One is overall reduction in yields, when crops do not fail but are less productive. Another is crop failure on a large scale. It appears that there is a trade-off in adjustment techniques for these two challenges.

\section{Conclusion}

Our results illustrate the complexity of climate change effects on rice yields at both the aggregate and individual levels, the scope of farmers' ability to counter climate change, and thus the importance of accurate modeling of farmers' decisions. Overall, farmers are unable to neutralize the adverse effects of the more extreme climate change. However, they are able

\footnotetext{
${ }^{17}$ Household's per capita income is compared to the province median per capita income of all households in our larger sample of 137 households in each of five years in the sample. Our results hold when we do comparisons using village-specific median per capita income, and also when we use per capita consumption in place of per capita income.

${ }_{18}^{18}$ Tables with results on soil quality and income are available in the web appendix.

${ }^{19}$ Recall that changes in yields predicted by DSSAT are driven solely by changes in climate and do not take into account any adjustments by farmers, while model predictions make adjustments to all production inputs according to the estimates of farmer's input decision rules.
} 
to cope with milder climate change and even benefit slightly from small increases in rainfall. While most farmers manage to adjust to milder climate change, poor farmers are less able to do so.

It should be noted that in our analysis we consider only farmers' adjustment through input decision rules. We do not model or incorporate possible changes in timing of input usage, nor broader adjustments such as changes in the type of crop grown and migration. As a result, our findings may overstate both yield changes and implied welfare effects of climate change.

\section{Acknowledgments}

We acknowledge support from the National Science Foundation, National Institute of Child Health and Human Development, and the Templeton Foundation, and thank Michael Greenstone, Andrew Foster, Jim Jones, Cheryl Porter, Gerrit Hoogenboom and Paul Wilkens for comments and help with the DSSAT and climate modeling.

\section{References}

Cruz, Rex Victor, Harasawa, Hideo, Lal, Murari, Wu, Shaohong, Anokhin, Yurij, Punsalmaa, Batima, Honda, Yasushi, Jafari, Mostafa, Li, Congxian, Ninh, Nguyen Huu. Asia. In: Parry, ML.Canziani, OF.Palutikof, JP.van der Linden, PJ., Hanson, CE., editors. Climate Change 2007: Impacts, Adaptation and Vulnerability. Contribution of Working Group II to the Fourth Assessment Report of the Intergovernmental Panel on Climate Change. Cambridge, UK: Cambridge University Press; 2007. p. 469-506.

Felkner, John, Tazhibayeva, Kamilya, Townsend, Robert M. An Integrated Economic-Crop Model of Multi-Stage Rice Production. Working paper, University of Chicago; 2009.

Fischer, Gunther, Shah, Mahendra, van Velthuizen, Harrij. Climate Change and Agricultural Vulnerability. Johannesburg: IIASA, Internat. Inst. for Applied Systems Analysis; 2002.

Giorgi, Filippo, Mearns, Linda O. Calculation of Average, Uncertainty Range, and Reliability of Regional Climate Changes from AOGCM Simulations Via the Reliability Ensemble Averaging (REA) Method. Journal of Climate. 2002; 15(10):1141-1158.

Nakicenovic, N., Alcamo, J., Davis, G., de Vries, B., Fenhann, J., Gaffin, S., Gregory, K., Grubler, A., Jung, TY., Kram, T. Special Report on Emissions Scenarios: A Special Report of Working Group III of the Intergovernmental Panel on Climate Change. New York: Cambridge University Press; 2000.

Parry M, Rosenzweig C, Iglesias A, Livermore M, Fischer G. Effects of Climate Change on Global Food Production under Sres Emissions and Socio-Economic Scenarios. Global Environmental Change. 2004; 14(1):53-67.

Richardson CW. Stochastic Simulation of Daily Precipitation, Temperature, and Solar Radiation. Water Resources Research. 1981; 17(1):182-190.

Rosenzweig C, Iglesias A, Yang XB, Epstein PR, Chivian, E E. Climate Change and Extreme Weather Events. Global Change \& Human Health. 2001; 2(2):90.

Thailand Database Research Archive. NORC/University of Chicago; 2009. http://.cier.uchicago.edu 


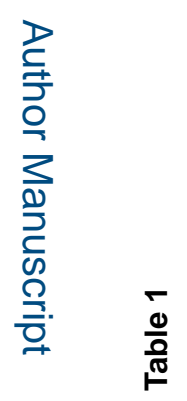

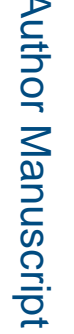

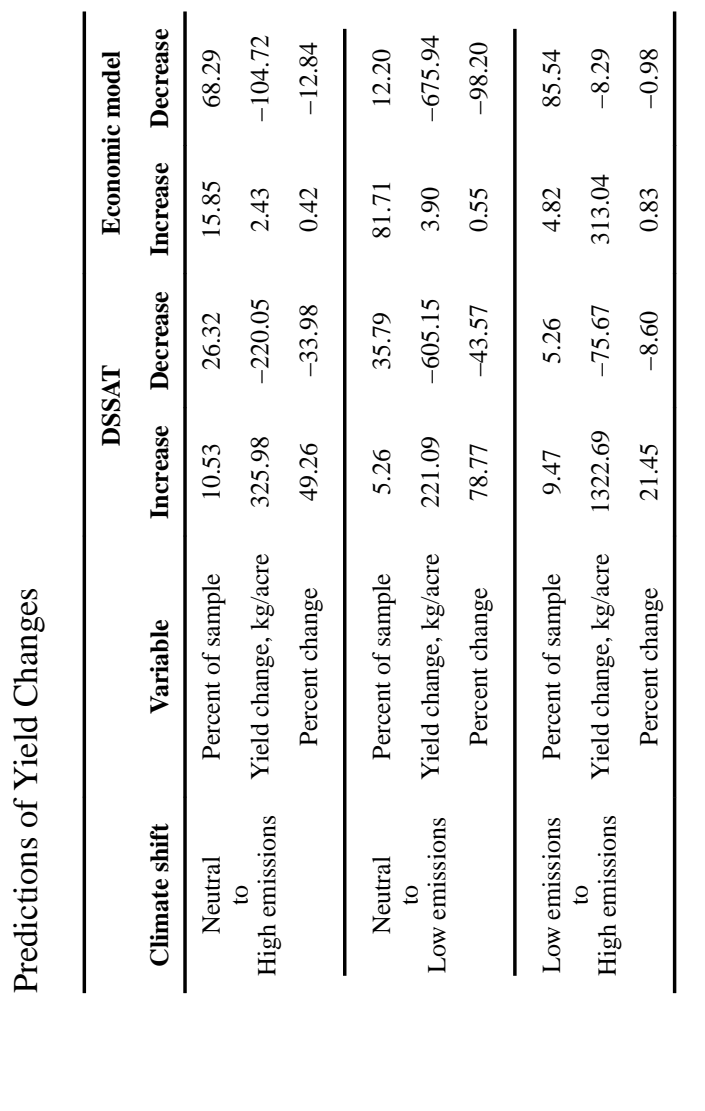

Am Econ Rev. Author manuscript; available in PMC 2010 May 01. 